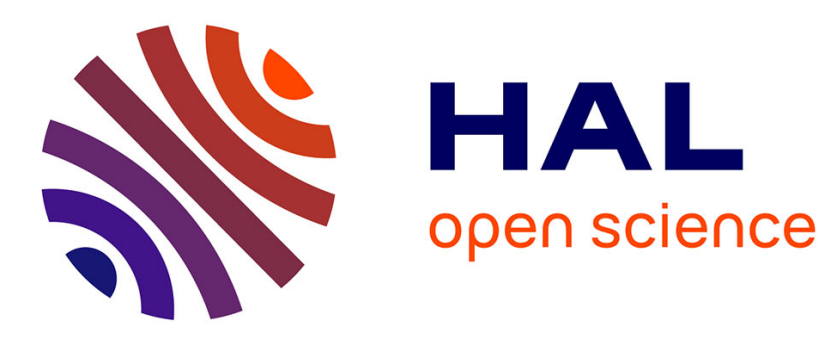

\title{
Contour tracking by minimal cost path approach. application to cephalometry
}

Barbara Romaniuk, Michel Desvignes, Marinette Revenu, Marie-Josèphe Deshayes

\section{- To cite this version:}

Barbara Romaniuk, Michel Desvignes, Marinette Revenu, Marie-Josèphe Deshayes. Contour tracking by minimal cost path approach. application to cephalometry. ICIP 2004, Oct 2004, Singapour, Singapore. pp.937-940, 10.1109/ICIP.2004.1419454 . hal-00807085

\section{HAL Id: hal-00807085 https://hal.science/hal-00807085}

Submitted on 2 Apr 2015

HAL is a multi-disciplinary open access archive for the deposit and dissemination of scientific research documents, whether they are published or not. The documents may come from teaching and research institutions in France or abroad, or from public or private research centers.
L'archive ouverte pluridisciplinaire HAL, est destinée au dépôt et à la diffusion de documents scientifiques de niveau recherche, publiés ou non, émanant des établissements d'enseignement et de recherche français ou étrangers, des laboratoires publics ou privés. 


\section{CONTOUR TRACKING BY MINIMAL COST PATH APPROACH. APPLICATION TO CEPHALOMETRY.}

\author{
B. Romaniuk ${ }^{1}$, M. Desvignes ${ }^{2}$, \\ ${ }^{1}$ GREYC Image \\ 6, Bvd Maréchal Juin \\ 14050 Caen Cedex, France \\ Barbara.Romaniuk@greyc.ismra.fr
}

\author{
M. Revenu ${ }^{1}$, M.-J. Deshayes \\ ${ }^{2}$ LIS-ENSERG \\ 961 rue de la Houille Blanche, BP 46 \\ 38402 Saint Martin d'Hères Cedex, France \\ michel.desvignes@lis.inpg.fr
}

\begin{abstract}
In this paper, a minimal cost approach is used for contour tracking with a good robustness. Dynamic programming was chosen for its efficiency. This general method is applied to the extraction of the cranial contour on high resolution X-Ray images. As a first step for automated localization of cephalometric points, an ellipse is then fitted on the extracted contour. This method was tested on 424 X-Ray images, with different acquisition parameters.
\end{abstract}

\section{INTRODUCTION}

In orthodontics, cephalometry is used as a therapeutic decision support and as a tool for early detection of dental dysharmonies. This method is based on the detection of particular anatomical points named cephalometric points. Studied points are often located on bones or/and sutures. A cephalometric analysis consists then in comparing angles and lengths obtained from the landmarked cephalometric points with corresponding normative values.

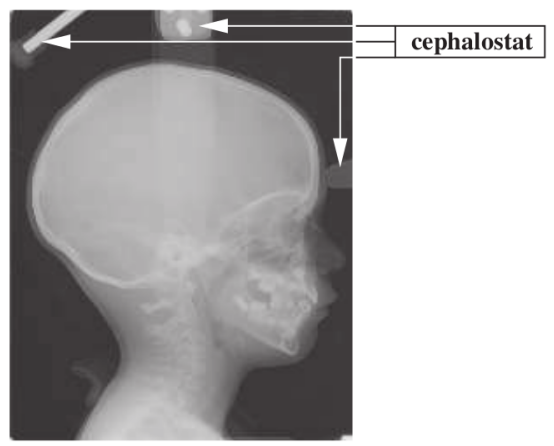

Fig. 1. X-ray image used in cephalometry.

In our case these points are identified on lateral X-ray images of the head, that are high resolution images (around 7 pixels for one millimeter). As in our former work [1], automation of cephalometric points landmarking is based on the detection and modeling of the cranial contour. We present here a method for detecting this contour that is based on the concept of minimal cost path and its subsequent modeling by an ellipse. This method was tested on 424 images acquired on different systems (Figure 1).

\section{METHOD}

The detection of the external cranial contour (dome of the skull limited by the nose and the lowest point of the cranium) by traditional methods used for image processing (gradients [2], active contours [3]) fails on a large database for some local configurations where intensity gradients are low or inverted. Moreover, these methods imply the setting of many parameters for a good detection of the contour (Figure 2). These parameters depend on the acquisition system and the quality of the image and robustness is affected.

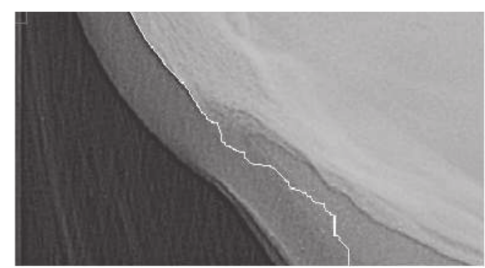

Fig. 2. Tracking with traditional methods.

In particular, low signal to noise ratio, low density of $\mathrm{X}$-rays, patient shape inter-variability and variable osseous density along this contour leads local methods to move away from the cranial contour, especially in presence of notches towards the interior of cranium or noise. Global methods like the Level Sets [4] or graph search methods have the disadvantage of a high algorithmic cost. Hence, we propose to use a regional approach based on a minimal cost path algorithm. We apply this method on the gradient image using 
the regularity of the image frame in order to combine robustness and low algorithmic cost. The adopted method is decomposed in three stages, followed by the modeling of the contour.

\subsection{Detection of two points on the cranial contour}

The first step of our method consists in automatically detecting two points on the cranial contour. This detection have to be independent on the source and quality of X-ray images. These two points are respectively localized in the front and in the back of the skull, in regions in which the cephalostat ${ }^{1}$ does not introduce supplementary information on the image. Two binary masks having the shape of arcs of circle are learned on a sample of images. They represent the former and the frontal parts of the cranium.

Let $I_{1}$ and $I_{2}$ be the two points that we want to detect. These points defined by practitioners and translated by image experts meet the criteria:

- stronger gradient

- location on the axis orthogonal to the mask

- location within less than 5 pixels from the point of maximum answer for the normalized correlation between the image and the binary masks.

Figure 3 presents the detection of $I_{1}$ and $I_{2}$.

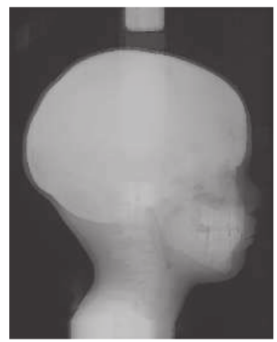

(a) Smoothed image

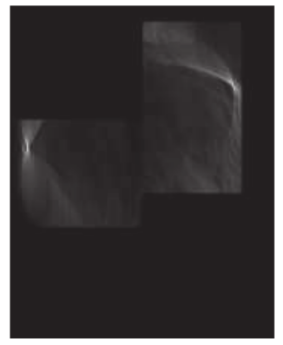

(b) Correlation with the masks

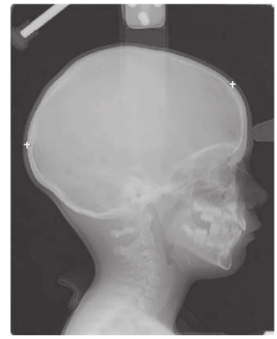

(c) Selected points
Fig. 3. Detection of two points on the cranial contour. (a) is the image on which we apply the masks; (b) is the normalized correlation between the image and the binary masks and (c) presents the detected points $I_{1}$ and $I_{2}$.

\footnotetext{
${ }^{1}$ part of the acquisition machine that allows a good positioning of the patient during the acquisition of the image (Figure 1).
}

\subsection{Contour tracking with a minimal cost path approach}

The following stage is the detection of the higher part of the cranial contour, between the two points $\mathrm{I}_{1}$ and $\mathrm{I}_{2}$ (step 1, Figure 4). An iterative approach is used for tracking this contour which is the stronger gradient path between $\mathrm{I}_{1}$ and $\mathrm{I}_{2}$. In this approach the best successor is computed at each iteration in a local region.

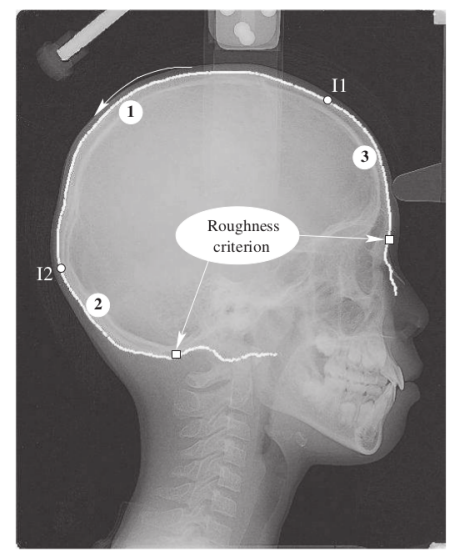

Fig. 4. Three parts of the contour

We seek the minimal cost path between points $C_{i}$ and $D_{i}$ defined by: $D_{i}=C_{i}+k \times T_{i}$, where $T_{i}$ is the general direction of the tangent to the path. Searching of the best successor is limited to the rectangle whose corners are $C_{i}$ and $D_{i}$ (Figure 5).

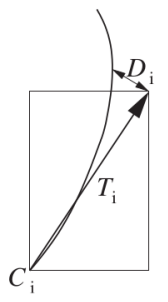

Fig. 5. Research of the best successor

Assuming the cranial contour is a monotonic curve in the rectangle $C_{i} D_{i}$, the shortest path can be iteratively and quickly computed by solving:

$$
C(x, y)=\min \left\{\begin{array}{l}
C(p r 1(x, y))+a\left(T_{i}\right) \times I(x, y) \\
C(p r 2(x, y))+b\left(T_{i}\right) \times I(x, y) \\
C(p r 3(x, y))+c\left(T_{i}\right) \times I(x, y)
\end{array}\right\}
$$

Functions $\operatorname{pr} 1(x, y), \operatorname{pr} 2(x, y)$ and $\operatorname{pr} 3(x, y)$ describe the predecessors of a point $(x, y)$ relatively to the general direction of the tangent $T_{i}$. Functions $a\left(T_{i}\right), b\left(T_{i}\right), c\left(T_{i}\right)$ are path lengths ( 1 or $\sqrt{2}$ ). The next point of the contour $C_{i+1}$ 
is then defined as the point located at a distance $d(d<k)$ from $C_{i}$ on this path. Tracking is then iterated from $C_{i+1}$.

The main idea here is to define a cost function. This function has to evaluate the cost of a contour by computing the cost for each point of this contour. This cost should be minimal at the starting point of an area. This minimal cost path is the contour that we search. The first points of this contour are retained and the tracking is iterated. The cost function must represent the contour intensity. It must also be invariant to contrast variations. The selected function is thus the opposite gradient of the image. The concept of regional maximum suppresses any concept of threshold and allows a great robustness. We consider that the detection of the higher part of the cranial contour is done when the path reaches the point $\mathrm{I}_{2}$.

We continue the tracking by detecting the lower parts of the cranial contour (steps 2 and 3, figure 4). The same tracking method is used, ending on a roughness criterion. We smooth the detected contour and compute the distance between the detected contour and the smoothed one. Let $\left\{\left(x_{i}, y_{i}\right)\right\}_{1<i<n}$ be the ordered set of points of the detected contour and $\left\{\left(x_{i}^{S}, y_{i}^{S}\right)\right\}_{1 \leq i \leq n}$ be the corresponding ordered set of points of the smoothed contour, smoothing being a simple mean operator. We consider here the euclidian distance:

$$
d\left(x_{i}, y_{i}\right)=\left(x_{i}-x_{i}^{S}\right)^{2}+\left(y_{i}-y_{i}^{S}\right)^{2} .
$$

In step 2 we aim to detect the point $(x, y)$ that is the frontier between the regular and the irregular part of the contour. This point verifies the criterion:

$$
\operatorname{argmax}_{i}\left(\sum_{i-\text { size }}^{i} d\left(x_{i}, y_{i}\right)-\sum_{i}^{i+s i z e} d\left(x_{i}, y_{i}\right)\right)
$$

where size is defined relatively to the size of the image.

In step 3 the goal is to find the first point presenting a hight irregularity. Let $(x, y)$ be this point and $M<n$ :

$$
(x, y) \in\left\{\left(x_{j}, y_{j}\right)\right\} \text {, with } y \leq y_{j} \forall j \in 1, \cdots, M,
$$

where $\left(x_{j}, y_{j}\right)$ are points corresponding to the $M$ greatest values in $\left\{d\left(x_{i}, y_{i}\right)_{i \in\{1, \cdots, n\}}\right\}$.

\subsection{Extrapolation of the contour by an ellipse}

The next step consists in the completion of the previously obtained contour by an ellipse. This can be addressed using a sample of points on each extremity of the contour. We can then compute an ellipse tangent to both extremities of the contour (Figure 6).

A conic section is represented by a set of points $(x, y)$ that verifies $F(\mathbf{a}, \mathbf{x})=0$, where $F$ is defined by:

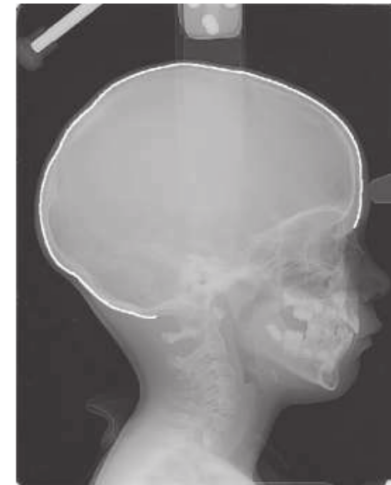

(a) First step: detection

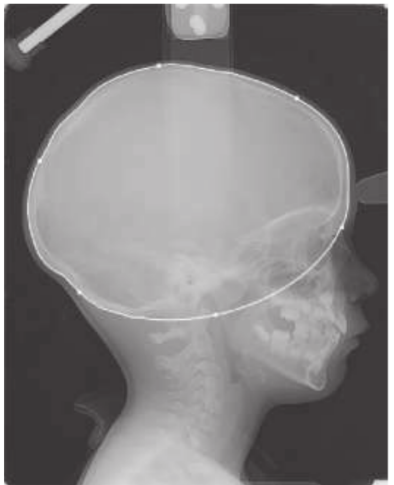

(b) Second step: completion
Fig. 6. Detection of the cranial contour in two steps.

$$
F(\mathbf{a}, \mathbf{x})=\mathbf{a x}=a x^{2}+b x y+c y^{2}+d x+e y+f,
$$

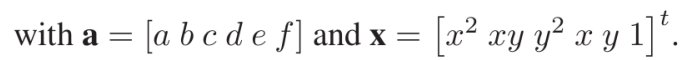

$F\left(\mathbf{a}, \mathbf{x}_{i}\right)$ is the algebraic distance from a point $\mathbf{x}_{i}$ to the conic section which equation is $F(\mathbf{a}, \mathbf{x})=0$. The problem is then to find for a set of $N$ points $\left\{\mathbf{x}_{1}, \mathbf{x}_{2}, \ldots, \mathbf{x}_{N}\right\}$ a vector â that verifies:

$$
\hat{\mathbf{a}}=\arg _{\mathrm{a}} \min \sum_{i=1}^{N} F\left(\mathbf{a}, \mathbf{x}_{i}\right)^{2} .
$$

Pilu et al. [5] pointed that using Lagrangian multipliers, the solution is given by the eigenvector associated to the single negative eigenvalue of the system:

$$
D^{t} D \mathbf{a}=S \mathbf{a}=\lambda C \mathbf{a}
$$

where $D=\left[\begin{array}{llll}\mathbf{x}_{1} & \mathbf{x}_{2} & \ldots & \mathbf{x}_{N}\end{array}\right]^{t}$ is the matrix bind to the data, $\lambda$ the eigenvalue associated to the eigenvector a and $C$ the matrix translating the algebraic constraint $b^{2}-4 a c=-1$ relative to the searched conic section.

$C$ is defined by:

$$
C=\left(\begin{array}{cccccc}
0 & 0 & 1 & 0 & 0 & 0 \\
0 & -2 & 0 & 0 & 0 & 0 \\
1 & 0 & 0 & 0 & 0 & 0 \\
0 & 0 & 0 & 0 & 0 & 0 \\
0 & 0 & 0 & 0 & 0 & 0 \\
0 & 0 & 0 & 0 & 0 & 0
\end{array}\right)
$$

We then have:

$$
b^{2}-4 a c=\mathbf{a}^{t} C \mathbf{a}=-1 .
$$

The shape of the matrix $C$ invites us to decompose the problem in blocks. We then define the matrices $S_{1}, S_{2}, S_{3}$, 
$S_{4}, \mathbf{a}_{1}, \mathbf{a}_{2}$ and $C_{1}$ from the generalized system (equation (6):

$$
\left\{\begin{array}{l}
S_{1} \mathbf{a}_{1}+S_{2} \mathbf{a}_{2}=\lambda C_{1} \mathbf{a}_{1} \\
S_{3} \mathbf{a}_{1}+S_{4} \mathbf{a}_{2}=0
\end{array}\right.
$$

Which can be rewritten as:

$$
\left\{\begin{aligned}
{\left[S-1+S_{2} S_{4}^{-1}\left(-S_{3}\right)\right] } & =\lambda C_{1} \mathbf{a}_{1} \\
\mathbf{a}_{2} & =S_{4}^{-1}\left(-S_{3} \mathbf{a}_{1}\right)
\end{aligned}\right.
$$

The inversion of the matrix $S_{4}$ is previously realized by Cholesky decomposition. We can then define a matrix $T$ as:

$$
T=\left[S-1+S_{2} S_{4}^{-1}\left(-S_{3}\right)\right] .
$$

Resolving the initial problem is then reduced to the resolution of $T \mathbf{a}_{1}=\lambda C_{1} \mathbf{a}_{1}$. It is eventually simplified to $T^{\prime} \mathbf{a}_{1}=\lambda \mathbf{a}_{1}$, with $T^{\prime}=C_{1}^{-1} T$. We define the characteristic polynomial of the system. The eigenvalue $\lambda$ is the smallest root of the characteristic polynomial of degree 3 . We can easily find a the eigenvector characterizing the unknown conic section.

The next step is to define a parametric equation of the ellipse from the implicit one. If a characterizes the ellipse, the equation:

$$
\mathbf{a x}=a x^{2}+b x y+c y^{2}+d x+e y+f=0
$$

is equivalent to:

$$
\left\{\begin{array}{l}
x=A \cos t \cos \alpha-B \sin t \sin \alpha+x_{0} \\
y=A \cos t \sin \alpha+B \sin t \cos \alpha+y_{0}
\end{array}, \quad t \in[0,2 \pi],\right.
$$

with

$$
\begin{gathered}
\alpha=\frac{\arctan \frac{b}{a-c}}{2}, x_{0}=\frac{2 d c-e b}{b^{2}-4 a c}, y_{0}=\frac{2 e a-d b}{b^{2}-4 a c}, \\
A=\sqrt{2 \frac{a x_{0}^{2}+c y_{0}^{2}+b x_{0} y_{0}-f}{a+c+\frac{b}{\sin 2 \alpha}}}, \\
B=\sqrt{2 \frac{a x_{0}^{2}+c y_{0}^{2}+b x_{0} y_{0}-f}{a+c-\frac{b}{\sin 2 \alpha}}} .
\end{gathered}
$$

\section{RESULTS}

We tested our method on 424 X-ray images of different quality. The detection of initial points $I_{1}$ and $I_{2}$ failed for two images. For the rest, the contour was correctly extracted in $97 \%$ of cases. Failures were principally due to a bad position of the subject during the acquisition: the back part of the skull is then out of the image. Figure 7 presents results obtained on 4 images with different resolution and quality and from different sources. Images (a) and (d) are numerical $\mathrm{X}$-ray images acquired on two different acquisition engines, (c) and (d) are scanned images.

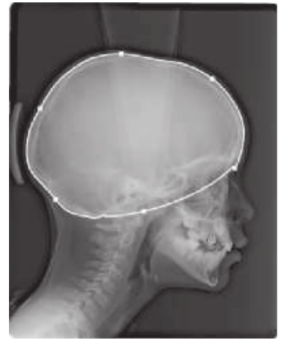

(a) Bad patients position

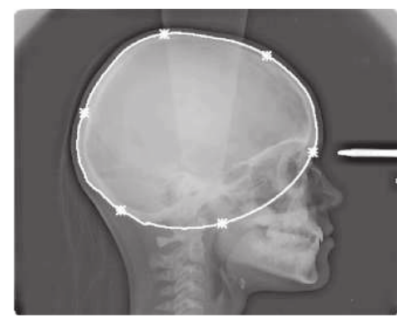

(c) Scanned image

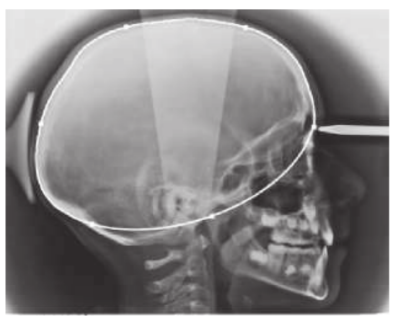

(b) Scanned image

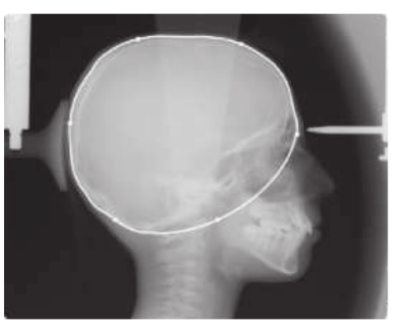

(d) Different source image
Fig. 7. Detection of the cranial contour on different sources and quality X-Ray images.

\section{CONCLUSION}

This paper presents an original, robust (different acquisition parameter or noise) and fast method for contour tracking which has been applied to cranial contour extraction. The detected contour can then be considered as the background for statistical localization of cephalometric points. It can be used to define an invariant coordinates space for the cephalometric problem. In our further work we proposed such a statistical non-linear modeling with an average precision of 2.5 millimeters.

\section{REFERENCES}

[1] M. Desvignes, B. Romaniuk, R. Demoment, M. Revenu, M.J. Deshayes Computer Assisted Landmarking of Cephalometric Radiographs. 4th IEEE SSIAI, pp. 296-300, April 2000.

[2] R. Deriche. Fast algorithms for low-level vision. IEEE Trans. on PAMI, Vol. 1(12), pp 78-88, 1990.

[3] P. Cocquerez, S. Philipp. Analyse d'images : filtrage et segmentation. Masson 1995.

[4] L.D. Cohen, R. Kimmel. Global Minimum for Active Contour Models: A Minimal Path Approach. IJCV, Vol. 24(1), pp. 57-78, August 1997.

[5] M. Pilu, A.W. Fitzgibbon, R.B. Fisher. Ellipse-specific Direct Least-square Fitting. ICIP, pp 599-603, 1996. 